\title{
Transduodenal ampullectomy in the treatment of villous adenomas and adenocarcinomas of the Vater's ampulla
}

\author{
J. A. Fraguela Mariña \\ General Surgery Department “A”. Complejo Hospitalario Universitario Juan Canalejo. A Coruña. Spain
}

\begin{abstract}
Introduction: adenomas are the most frequent tumors of the Vater's ampulla. Their capacity for malignant transformation following the adenoma-carcinoma sequence is well known. It is because of this that resection after diagnosis is required. The identification of the appropriate technique according to tumor features would require that patients not be undertreated or overtreated, which would give rise to serious consequences derived from their location.

Patients and methods: villous adenomas and adenocarcinomas of the Vater's ampulla candidates for local resection were revised from January 1st, 1998 through June $30^{\text {th }}, 2003$. We describe the methods of diagnosis and ampulectomy techniques we performed.

Results: we performed an ampulectomy by first intention in all 8 patients included in this study. However, pancreatoduodenectomy was necessary in two patients because of the closeness of resection margins. We had no mortality in this series, and morbidity was limited to two episodes of digestive bleeding that were controlled by electrocoagulation and embolization. The mean follow-up was 28.5 months (range, 6-72 months).

Conclusions: the difficulty of precise preoperatory diagnosis in adenomas of the Vater's ampulla demands resection after identification. Ampulectomy is the treatment of choice for villous adenomas and $\mathrm{T} 1$ adenocarcinomas, with $1 \mathrm{~cm}$ of resection margin to avoid local recurrence.
\end{abstract}

Key words: Vater's ampulla. Papillary tumor. Ampullectomy.

Fraguela Mariña JA. Transduodenal ampullectomy in the treatment of villous adenomas and adenocarcinomas of the Vater's ampulla. Rev Esp Enferm Dig 2003; 96: 829-834.

Recibido: 13-01-04.

Aceptado: 09-03-04.

Correspondencia: José Antonio Fraguela Mariña. C/ General Sanjurjo, 48, portal 3, 4 D. 15006 A Coruña. Tel.: 981178 166. e-mail: jafm@canalejo.org

\section{INTRODUCTION}

In 1720, Abraham Vater described a new anatomic entity that today is called the Vater's ampulla (1). It is formed in most individuals by the union of the terminal segments of the pancreatic and common bile ducts. In $25 \%$ of individuals, the ampulla is formed by the ending of the common duct, having the Wirsung's duct its own entry point into the duodenum just adjacent to the ampulla. The ampulla opens into the duodenum through a small mucosal elevation known as the papilla of Vater. Tumors of the Vater's ampulla are called ampulomas and represent $2 \%$ of all the tumors of the gastrointestinal tract (2). The two most usual tumors are adenomas (about $70 \%$ villous adenomas) and adenocarcinomas $(2,3)$.

The aim of this study is to attempt to identify patients that would benefit from transduodenal ampullectomy in the treatment of ampullary tumors, showing our experience in surgical ampullectomy in the treatment of villous adenomas and adenocarcinomas of the ampulla of Vater.

\section{PATIENTS AND METHODS}

Patients with villous adenomas and adenocarcinomas of the ampulla of Vater liable to local transduodenal resection and undergoing ampullectomy were retrospectively reviewed from January 1st, 1998 to June $30^{\text {th }}, 2003$ within the General Surgical Department "A" of the "Juan Canalejo Hospital", A Coruña, Spain. In that period of time, 28 patients underwent surgery with a diagnosis of adenocarcinoma of the Vater's ampulla and three villous adenomas of the papilla, one of them associated with an adenocarcinoma. Eight patients were treated by transduodenal ampullectomy, five males and three females. In the remaining patients we performed a pancreatoduodenectomy (PD). The mean age at presentation for patients undergoing transduodenal ampullectomy was 67 years 
(52-78 years). The two most common presenting symptoms were abdominal pain, in 5 cases, and jaundice, in 3. The presence of these symptoms led us to perform hepatic function tests and an abdominal ultrasonography. Endoscopic retrograde cholangiography (ERCP) showed a tumor of the ampulla in all cases, and biopsy confirmed the diagnosis. An abdominal computed tomography (CT) was further performed in all patients, and carcinoembryonic antigen (CEA) levels were measured. None of them was studied by endoscopic ultrasonography.

All of our patients were operated on by a right subcostal incision and, after cholecystectomy, the biliary duct was catheterized with a Fogarty catheter through the cystic duct in order to identify the papilla. After vertical duodenotomy a submucosal injection of 1/100.000 adrenaline was administered in order to avoid bleeding, and a resection of the papilla was made leaving $1-\mathrm{cm}$ margins in all levels, including the pancreatic margin. After that, the bile duct and the pancreatic duct were reinserted with interrupted synthetic absorbable sutures (5-0), and then the duodenotomy was closed. Frozen sections of the resection margins were obtained during surgery. If the tumor was close to the resection margin, a PD was performed as a Whipple's procedure. Locoregional lymph node dissection was performed only after PD.

\section{RESULTS}

Hepatic function tests showed cholestasis in all cases. A dilatation of the bile duct was demonstrated in all patients by ultrasonography, but its origin was not identified. CEA was always normal, and the extension study by CT was normal as well. ERCP was diagnostic in $100 \%$ of cases, and the diagnostic accuracy of biopsy was correct in two patients with villous adenomas and in five with adenocarcinomas; a false negative diagnosis of villous adenoma was made in a patient with an adenocarcinoma.

All patients underwent ampullectomy; the tumor was near the resection margin in 3 of 6 adenocarcinomas, and surgery was completed by means of PD in two cases; the third patient was a poor candidate for this type of surgery; therefore, the operation was not completed, and the pa- tient died 13 months later. All patients were informed on the possibility of a PD; six of them agreed, but a patient with a high surgical risk refused, as did another patient with an initial diagnosis of villous adenoma in whom, resection confirmed the presence of an adenocarcinoma.

None of the patients died, but two patients developed upper gastrointestinal bleeding (UGB), which were solved by endoscopic electrocoagulation 24 hours after surgery in one patient, and by embolization of the gastroduodenal artery in the other one.

Follow-up ranged from 6 to 72 months. One patient with PD because of a resection margin close to a moderately differentiated tumor showed 58 months after surgery a metastasis at the abdominal wall, which was resected; 12 months later a new metastasis in the right iliac chain with compression of the crural nerve was also removed. The other patients remained without symptoms (Table I).

\section{DISCUSSION}

Ampullary tumors include a wide variety of pathological entities. Adenoma is the most frequent tumor, and its progression to villous adenoma and adenocarcinoma is admitted, much in the same way as the adenoma-carcinoma sequence in colon polyps. Villous adenomas of the Vater's ampulla are considered premalignant lesions, their resection being indicated to prevent the development of adenocarcinomas, which takes place in 25 to $40 \%$ of patients. However, $40 \%$ of cases are "in situ" carcinomas $(4,5)$, as it was the case in one of our three patients $(33 \%)$. There is no single treatment desirable for all lesions; moreover, not all adenocarcinomas require $\mathrm{PD}$, and not all benign lesions are suitable for local resection (5). An appropriate selection of those patients in whom local resection would be safe and effective would allow this suitable treatment, depending on the kind of tumor.

The pathological characteristics of the papilla and the difficulties of biopsy sampling make a histologically accurate diagnosis at the time of endoscopy not an easy task. Macrobiopsies and a brushing cytology help in diagnostic accuracy, but a suitable classification requires a

Table I

\begin{tabular}{|c|c|c|c|c|c|c|}
\hline Case & Sex & Age & Diagnosis & Procedure & Complications & Follow-up \\
\hline 1 & $\mathrm{~F}$ & 52 & Adenocarcinoma & Ampullectomy + PD & - & 72 months \\
\hline 2 & $\mathrm{~F}$ & 72 & Villous adenoma & Ampullectomy & - & 60 months \\
\hline 3 & $\mathrm{~F}$ & 57 & Villous adenoma & Ampullectomy & UGB & 36 months \\
\hline 4 & $\mathrm{M}$ & 77 & Adenocarcinoma & Ampullectomy & - & 17 months \\
\hline 5 & $\mathrm{M}$ & 72 & Adenocarcinoma & Ampullectomy & UGB & 14 months \\
\hline 6 & M & 78 & Adenocarcinoma & Ampullectomy & - & 13 months $(+)$ \\
\hline 7 & $\mathrm{M}$ & 70 & Villous+Adenoca. & Ampullectomy & - & 8 months \\
\hline 8 & $\mathrm{~F}$ & 65 & Adenocarcinoma & Ampullectomy + PD & - & 6 months \\
\hline
\end{tabular}


complete resection of the tumor and its histological examination, since the presence of an "in situ" or infiltrating carcinoma within an adenoma cannot be excluded without complete resection (5-7). In our series, only one patient (case 7) was first diagnosed as having a benign tumor and then turned out to reveal a malignant lesion after total resection, which confers us a diagnostic accuracy of $87.5 \%$. Endoscopy and cholangioresonance, performed before sphincterotomy and endoprosthesis insertion to minimize interpretation mistakes, allow lesion staging, overall and regarding depth of penetration into the duodenal wall and the pancreas. However, overstaging due to submucosal edema from associated pancreatitis has been reported in as much as one third of lesions. Both techniques are less helpful in accurately determining the presence or absence of lymph node metastases $(8,9)$. However, they would be able to select patients who may be optimally managed by endoscopic resection. In our patients we performed an extension study by means of CT to identify parameters against surgery. Biopsy at the time of surgery with a total resection of the lesion allowed a final diagnosis and clearly revealed the depth of tumor penetration into the wall, with no problems regarding biopsy interpretation in patients with a previous papillotomy (6), perhaps because all patients underwent surgery 3 to 7 days after papillotomy, thus minimizing interpretation errors due to regenerative changes.

It is considered nowadays that all ampulla tumors should be resected because of their risk of malignant transformation (10). Controversy arises as to what kind of resection is suitable for each tumor and each patient.

Ampullectomy was first described by Halsted in 1899 (11). After a golden age at the beginning of the last century, it was progressively abandoned due to postoperative complications and mortality, a high rate of recurrences, and the introduction of PD. Nowadays it is associated with a lower rate of morbidity and mortality, and with less hospitalizations than PD $(5,7,12)$; moreover, local recurrence rates are low if indication and surgical technique are suitable, thus allowing a safe resection margin of 1 $\mathrm{cm}$ (7) in adenocarcinomas and quite lower in villous adenomas. Endoscopic snare resection does not allow a wide resectional margin on the pancreatic side, and the possibilities of local recurrence are higher, which restricts its indication as a palliative treatment for patients unfit for surgery or rejecting it (13). A wide series showed that the most important prognostic factor for survival was complete tumor resection (14-16). In villous adenomas and adenocarcinomas, pT1 ampullectomy results in the same, or even better, overall outcomes than PD $(7,17)$. We performed it in 6 patients, and a follow-up between 8 and 60 months showed no evidence of recurrence. However, an episode of upper gastrointestinal bleeding occurred in two patients. This has been reported as a complication quite often (10) probably because of the use of adrenaline, which makes the resection easier but may result in rebleeding once metabolized. When the resection margin was affected or close to the tumor, which usually occurs with poorly or moderately differentiated tumors, we performed a PD with a previous informed consent of the patients. Case 7 refused to go on with PD if there was an indication for that procedure; so, although the resection margin was lower than $1 \mathrm{~cm}$, we performed only an ampullectomy. Eight months after being operated on he remains without symptoms, and had no signs of recurrence during an endoscopy performed at 6 months.

The Vater's ampulla has a distinct pattern of lymphatic drainage, and in contrast to pancreatic tumors, ampullary tumors spread into the nearby retroduodenal nodes, even in non-advanced cases. Because of this, some authors advocate for both local lymphadenectomy and ampullectomy in order to improve prognosis $(18,19)$. Only when local nodes are involved in T1 poorly differentiated tumors with suitable resectional margins, PD would be justified as a complementary treatment (7). For T2 well differentiated tumors with suitable resection margins and no nodal involvement, ampullectomy would be probably chosen. Pancreatic involvement will lead to PD with lymphadenectomy, because of lymphatic spread after pancreatic dissemination, as it happened in two of our patients.

Endoscopic follow-up after ampullectomy is important, and we performed endoscopy every six months during the first three years, and yearly thereafter in order to detect recurrences that might lead to PD.

To conclude, we may say that transduodenal ampullectomy is an accurate treatment for villous adenomas of the papilla if resection is complete, and it may be a safe treatment for well-differentiated adenocarcinomas whenever we succeed in having a safe resection margin of at least $1 \mathrm{~cm}$ at all levels.

\section{REFERENCES}

1. Vater A. Disertatio anatomica qua novum bilis diverticulum circa orificium ductus choledochi, at et valvulosum colli vesical fellae constructionem ad discepterdum proponit. Wittenberg, Lit Gerdesianis, 1720. p. 2-32

2. Seifert W, Schulte F, Stolte M. Adenoma and carcinoma of the duodenum and papilla of Vater: a clinicopathologic study. Am J Gastroenterol 1992; 87: 37-41.

3. Sharp KW, Brandes JL. Local resection of tumors of the ampulla of Vater. Am Surg 1990; 56: 214-7.

4. Seifert E, Schulte F, Stolte M. Adenoma and carcinoma of the duodenum and papilla of Vater: a clinicopathologic study. Am J Gastroenterol 1992; 87: 37-42.

5. Rattner DX, Fernández C, Brugger WR, Warshaw AL. Defining the criteria for local resection of ampullary neoplasms. Arch Surg 1996; 131: 366-71.

6. Rodríguez C, Borda F, Elizalde I, Jiménez FJ, Carral D. ¿Hasta qué punto es seguro el diagnóstico preoperatorio de la biopsia endoscópica en el tumor ampular? Rev Esp Enferm Dig 2002; 94 (10): 585-8.

7. Beger H, Treitschke F, Gansauge F, Harada H, Hiki N, Mattfeldt T. Tumor of the ampulla of Vater. Experience with local or radical resection in 171 consecutively treated patients. Arch Surg 1999; 134: 526-32.

8. Souquet JC, Napoleon B, Pujol B, Ponchon T, Keriven O, Lambert R. Ecoendoscopy prior to endoscopy tumor therapy: more safety? Endoscopy $1993 ; 25$ : 475-8. 
9. Géier A, Nguyen HN, Gartung C, Matern S. MRCP and ERCP to detect small ampullary carcinoma. Lancet 2000; 356 (9241): 1607-8.

10. Chareton B, Coiffic J, Landen S, Bardaxoglou E, Campion JP, Launois B. Diagnosis and therapy for ampullary tumors: 63 cases. World J Surg 1996; 20: 707-12.

11. Halsted W. Contributions to the surgery of the bile passages especially of the common bile duct. Boston Med Surg 1889; 141: 64554.

12. Farouk M, Niotis M, Branum G, Cotton PB, Meyers WC. Indications for and the technique of local resection of tumors of the papila of Vater. Arch Surg 1991; 126: 650-2.

13. Tokunaga Y, Hosogi H, Hoppou T, Nagakami M, Tokuka A, Ohsumi $\mathrm{K}$. A case of ampullary carcinoma successfully managed with endoscopic snare resection. Surg Laparosc Endosc Percutan Tech 2002; 12 (4): $273-8$.
14. Monson JRT, Donohue JH, McEntee GP. Radical resection for carcinoma of the ampulla of Vater. Arch Surg 1991; 126: 353-7.

15. Matory YL, Gaynor J, Brennan M. Carcinoma of the ampulla of Vater. Surg Gynecol Obstet 1993; 177: 366-70.

16. Allema JH, Reinders ME, van Gulik TM. Results of pancreaticoduodenectomy for ampullary carcinoma and analysis of prognostic factors for survival. Surgery 1995; 117: 247-53.

17. Asbun HJ, Rossi RL, Munson Jl. Local resection for ampullary tumors: is there a place for it? Arch Surg 1993; 128: 515-20.

18. Hanyu F, Imaizumi T, Nakamura M, Yoshikawa T. Proposed definition of early cancer of the papilla of Vater. J Biliary Tract Pancreas 1984; 5: 847-52.

19. Willet CG, Warshaw AL, Convery K, Compton CC. Patterns of faillure after pancreaticoduodenectomy for ampullary carcinoma. Surg Gynecol Obstet 1993; 176: 33-8.

\title{
Ampulectomía transduodenal en el tratamiento de los adenomas vellosos y adenocarcinomas de la ampolla de Vater
}

\author{
J. A. Fraguela Mariña \\ Servicio de Cirugía General y del Aparato Digestivo “A”. Complejo Hospitalario Universitario Juan Canalejo. A Coruña
}

\section{RESUMEN}

Introducción: los tumores más frecuentes de la ampolla de Vater son los adenomas. Es conocida su capacidad de malignización mediante la secuencia adenoma-carcinoma, por lo que requieren su resección, tras su diagnóstico. El establecimiento de la técnica adecuada según las características del tumor permitiría no infratratar o sobretratar pacientes, con las graves consecuencias que se derivan de ello, debido a su localización.

Pacientes y métodos: se revisan los adenomas vellosos y adenocarcinomas de ampolla de Vater subsidiarios de resección local desde el 1 de enero de 1998 al 30 de junio de 2003. Se describen los métodos diagnósticos y la técnica de ampulectomía empleada.

Resultados: en los 8 pacientes estudiados se realizó ampulectomía de primera intención, aunque tuvo que completarse con duodenopancreatectomía cefálica en dos casos debido a la proximidad del borde de resección. No existió mortalidad en la serie y la morbilidad se limitó a dos episodios de HDA que fueron controlados mediante electrocoagulación y embolización. El seguimiento medio fue de 28,5 meses (6-72).

Conclusiones: la dificultad del diagnóstico preoperatorio preciso en los adenomas de la ampolla de Vater exige la resección tras su diagnóstico. La ampulectomía es el tratamiento de elección en los adenomas vellosos y adenocarcinomas T1 si se consigue un margen de resección de $1 \mathrm{~cm}$, evitándose la recidiva local.

Palabras clave: Papila de Vater. Tumor papilar. Ampulectomía.

\section{INTRODUCCIÓN}

En 1720 Abraham Vater describió una nueva entidad anatómica que hoy reconocemos como ampolla de Vater (1). Está formada en la mayoría de los individuos por la unión de los segmentos terminales del conducto pancreático y del colédoco. En el $25 \%$ de los casos la ampolla está formada sólo por la terminación del colédoco, teniendo el Wirsung su propia entrada en el duodeno independiente y adyacente a la ampolla. La ampolla se abre en el duodeno a través de una pequeña elevación mucosa que conocemos como papila de Vater. Los tumores de la ampolla de Vater se denominan ampulomas y representan el $2 \%$ de todos los tumores del tracto digestivo (2). Los dos tipos más habituales de tumores son los adenomas (en torno al 70\% adenomas vellosos) y los adenocarcinomas $(2,3)$.

El objetivo de este trabajo es intentar identificar los pacientes que se beneficiarían de una ampulectomía transduodenal en el tratamiento de los tumores ampulares reflejando nuestra experiencia en la ampulectomía quirúrgica en el tratamiento de adenomas vellosos y adenocarcinomas de la ampolla de Vater. 


\section{PACIENTES Y MÉTODOS}

Se realiza una revisión retrospectiva de los pacientes con adenomas vellosos y adenocarcinomas de la ampolla de Vater susceptibles de exéresis local transduodenal y sometidos a ampulectomía entre el 1 de enero de 1998 y el 30 de junio de 2003 en el Servicio de Cirugía General y del Aparato Digestivo "A" del Hospital "Juan Canalejo" de A Coruña, España. Se han intervenido en dicho periodo 28 pacientes con diagnóstico anatomopatológico de adenocarcinoma de la ampolla de Vater y tres adenomas vellosos de la papila, uno de ellos asociado a adenocarcinoma. Hemos tratado mediante ampulectomía transduodenal a 8 pacientes, 5 varones y 3 hembras. El resto fueron sometidos a DPC (duodenopancreatectomía cefálica). La edad media de presentación de los pacientes sometidos a ampulectomía transduodenal fue de 67 años (rango 52 a 78). El síntoma más frecuente fue el dolor abdominal, en 5 casos, seguido de ictericia en 3. La presencia de dichos síntomas condujo a la realización de una bioquímica hepática, y una ecografía abdominal. La colangiopancreatografía retrógrada endoscópica (CPRE) evidenció la presencia de una tumoración de la ampolla y la biopsia de dicha tumoración nos orientó al diagnóstico. En todos los pacientes se realizó tomografía computerizada (TC) abdominal como estudio de extensión y se estudiaron los niveles de antígeno carcinoembrionario (CEA). Ningún paciente fue estadificado mediante ecoendoscopia.

Todos los pacientes fueron intervenidos mediante una incisión subcostal derecha y tras realizar colecistectomía, se cateterizó con un Fogarty la vía biliar a través del cístico para identificar la papila. Tras una duodenotomía vertical se efectuó una inyección submucosa de solución de adrenalina a 1/100.000 para evitar el sangrado y se procedió a la exéresis de la papila dejando un $\mathrm{cm}$ de margen en todos los planos, incluido el pancreático. Posteriormente se procedió a reimplantar el colédoco y el conducto pancreático con sutura reabsorbible de 5/0 realizándose a continuación el cierre de la duodenotomía. El espécimen obtenido fue enviado para estudio anatomopatológico intraoperatorio para valorar los bordes de resección. Si el tumor estaba próximo al borde de resección se realizó DPC según la técnica de Whipple. Sólo se realizó linfadenectomía locorregional tras DPC.

\section{RESULTADOS}

La bioquímica hepática evidenció un patrón colostásico en todos los casos. La ecografia abdominal permitió identificar dilatación de la vía biliar intra y/o extrahepática en todos los enfermos sin evidenciar nunca la causa. El CEA siempre fue normal y la TC nunca reveló la presencia de extensión de la enfermedad. La ERCP fue diagnóstica en el $100 \%$ de los casos y el diagnóstico biópsico fue correcto en dos adenomas vellosos y 5 adenocarcinomas, identificándose un falso negativo de adenoma velloso que resultó presentar componentes de adenocarcinoma.

En todos los pacientes se realizó ampulectomía. Se apreció un tumor próximo al borde de resección en 3 de los 6 adenocarcinomas. Se completó la cirugía mediante DPC en 2 de estos casos. Por ser el tercer paciente un mal candidato para dicha cirugía, no se prosiguió con la intervención y falleció a los 13 meses. Todos los pacientes fueron informados de la posibilidad de continuar con una DPC si existían datos para completar la intervención. Seis de ellos lo aceptaron pero lo rechazó un pacientes en el que el riesgo quirúrgico era alto y otro en el que el diagnóstico inicial era de adenoma velloso pero cuya exéresis confirmó la presencia de un adenocarcinoma.

La mortalidad fue nula y la morbilidad fue de 2 pacientes con hemorragia digestiva alta (HDA) que se solucionaron mediante electrocoagulación endoscópica a las 24 horas en un paciente y por embolización de la arteria gastroduodenal en el otro.

El seguimiento abarca desde los 6 a los 72 meses. Una paciente a la que se realizó DPC por borde de resección de tumor moderadamente diferenciado muy próximo al tumor, presentó a los 58 meses una metástasis en la pared abdominal de la que fue intervenida y requirió nueva intervención por metástasis en la cadena iliaca derecha con compresión del nervio crural de la que fue intervenida 12 meses después. El resto de los pacientes permanecen asintomáticos (Tabla I).

\section{DISCUSIÓN}

Los tumores ampulares representan una amplia variedad de entidades patológicas. El tumor más frecuente es

Tabla I

\begin{tabular}{|c|c|c|c|c|c|c|}
\hline Caso & Sexo & Edad & Diagnóstico & Intervención & Complicaciones & Seguimiento \\
\hline 1 & $\mathrm{H}$ & 52 & Adenocarcinoma & Ampulectomía + DPC & - & 72 meses \\
\hline 2 & $\mathrm{H}$ & 72 & Adenoma velloso & Ampulectomía & - & 60 meses \\
\hline 3 & $\mathrm{H}$ & 57 & Adenoma velloso & Ampulectomía & HDA & 36 meses \\
\hline 4 & V & 77 & Adenocarcinoma & Ampulectomía & - & 17 meses \\
\hline 5 & V & 72 & Adenocarcinoma & Ampulectomía & HDA & 14 meses \\
\hline 6 & V & 78 & Adenocarcinoma & Ampulectomía & - & 13 meses $(+)$ \\
\hline 7 & V & 70 & Velloso+adenoca. & Ampulectomía & - & 8 meses \\
\hline 8 & $\mathrm{H}$ & 65 & Adenocarcinoma & Ampulectomía + DPC & - & 6 meses \\
\hline
\end{tabular}


el adenoma y su progresión a adenoma velloso y adenocarcinoma está admitida en el mismo sentido que la secuencia adenoma-carcinoma de los pólipos de colon. Los adenomas vellosos de la papila de Vater son considerados lesiones premalignas. Por ello que está indicada su exéresis con el fin de evitar el desarrollo de adenocarcinoma. Esto acontece en el 25 al $40 \%$ de los pacientes, aunque en el $40 \%$ de los casos se trata de carcinomas son "in situ" 4,5 , como sucedió en uno de nuestros tres casos (33\%). No existe un único tratamiento deseable para todas las lesiones y además, no todos los adenocarcinomas requieren DPC ni todas las lesiones benignas se beneficiarán de resección local (5). La selección adecuada de los pacientes en los que una resección local fuera segura y eficaz, permitiría un tratamiento apropiado según el tipo de tumor.

Las características anatomopatológicas de la papila y las limitaciones en la toma de biopsia, hace que el diagnóstico histológico preoperatorio correcto mediante endoscopia no sea sencillo ni fiable. La realización de macrobiopsias y citología por cepillado ayudan en la precisión diagnóstica; pero su adecuada catalogación exige la resección completa del tumor y su biopsia extemporánea, ya que es imposible valorar con certeza en cada paciente la presencia de un carcinoma in situ o un carcinoma infiltrante en los adenomas sin su excisión completa (5-7). En nuestra serie sólo un paciente (caso 7) fue diagnosticado inicialmente como benigno, confirmándose la malignidad tras la exéresis total del tumor, lo que confiere una precisión diagnóstica del 87,5\%. La ecoendoscopia y la colangiorresonancia, realizadas previamente a la esfinterotomía y a la inserción de una endoprótesis para minimizar errores de interpretación, permiten el estadiaje de la lesión, sobre todo en cuanto al grado de penetración en la pared y en el páncreas, aunque el sobreestadiaje debido a edema de la submucosa por pancreatitis puede llegar a ocurrir en un tercio de las lesiones. Ambas técnicas son menos fiables a la hora de establecer el grado de afectación ganglionar $(8,9)$, aunque podrían identificar a los pacientes que se beneficiarían de una resección endoscópica. En nuestros pacientes realizamos estudio de extensión mediante TC para identificar criterios que contraindicaran la intervención. La biopsia intraoperatoria con exéresis total de la lesión nos permitió establecer el diagnóstico definitivo y el grado de penetración tumoral en la pared sin que haya habido problemas de interpretación de las biopsias en pacientes con papilotomía previa (6), quizás porque todos los pacientes fueron intervenidos entre 3 y 7 días tras la realización de la misma con lo que se minimizan los errores de interpretación debidos a los cambios regenerativos.

Actualmente se considera que todos los tumores ampulares deben ser resecados debido al riesgo de malignización (10). La controversia surge al intentar establecer qué tipo de resección es la adecuada para las características del tumor y del paciente.

La ampulectomía fue descrita por primera vez por Halsted en 1899 (11). Tras su época dorada a principios de siglo, fue progresivamente abandonada debido a su morbimortalidad postoperatoria, su alta tasa de recidivas y la introducción de la DPC. Actualmente se asocia con menor morbi- mortalidad y menor estancia hospitalaria que la DPC $(5,7,12)$, así como escasa tasa de recidivas si la indicación y la técnica quirúrgica se establecen correctamente, dejando un margen de resección de $1 \mathrm{~cm}$ (7) en caso de adenocarcinomas y algo menor en caso de adenomas vellosos. La escisión endoscópica local no permite un margen amplio de resección del borde pancreático, por lo que las posibilidades de recidiva local serían altas, limitándose por tanto su indicación como tratamiento paliativo de pacientes inapropiados para cirugía o que rechazan la intervención (13). En largas series se demostró que el factor pronóstico más importante para la supervivencia es la resección completa del tumor (14-16). En adenomas vellosos y adenocarcinomas pT1, la ampulectomía tiene igual o mejores resultados globales que la DPC $(7,17)$. Nosotros la hemos realizado en 6 pacientes con un seguimiento entre 8 y 60 meses sin signos de recidiva, aunque en dos pacientes se ha producido un episodio de HDA. Esta complicación está descrita como complicación con cierta frecuencia (10), secundaria, probablemente al uso de adrenalina, que aunque facilita la exéresis, posibilita el resangrado tras ser metabolizada. Cuando el borde de resección estaba afectado o muy próximo al tumor, que suele coincidir con tumores poco o moderadamente diferenciados, procedimos a DPC previo consentimiento informado del paciente establecido antes de la cirugía. El paciente correspondiente al caso 7 se negó a proseguir con la DPC si existía indicación para la misma, por lo que, a pesar del que el margen de resección era menor a $1 \mathrm{~cm}$, se realizó simplemente ampulectomía. A los 8 meses de la intervención permanece asintomático sin signos de recidiva en la endoscopia realizada a los 6 meses.

La ampolla de Vater tiene un patrón de drenaje linfático distinto a los tumores de páncreas, afectando a los ganglios próximos retroduodenales, incluso en casos avanzados, por lo que algunos autores abogan por la linfadenectomía local junto con la ampulectomía para mejorar el pronóstico $(18,19)$. Sólo la afectación de ganglios locales en tumores $\mathrm{T} 1$ poco diferenciados con margen de resección aceptable, justificarían completar la intervención con DPC (7). En tumores T2 bien diferenciados con margen de resección aceptable sin afectación ganglionar, probablemente la ampulectomía sería así mismo suficiente. La afectación pancreática obligaría a DPC con linfadenectomía locorregional, debido a las características de diseminación linfática tras afectación pancreática como sucedió en dos de nuestros pacientes.

Es importante el seguimiento endoscópico tras ampulectomía, que nosotros realizamos cada 6 meses durante los tres primeros años, y posteriormente con periodicidad anual, para detectar recidivas que obliguen a la realización de DPC.

En conclusión, podemos decir que la ampulectomía transduodenal es el tratamiento adecuado para adenomas vellosos de papila siempre que la exéresis sea completa y podría ser el tratamiento ideal para adenocarcinomas de la papila bien diferenciados, siempre que se consiga un margen de resección en todos los planos de al menos $1 \mathrm{~cm}$. 